\title{
Assessing distribution network hosting capacity with the addition of soft open points
}

\author{
L J Thomas*, A Burchill*, D J Rogers ${ }^{\dagger}$, M Guest*, N Jenkins* \\ ${ }^{\star}$ Cardiff University,UK-ThomasL62@cf.ac.uk,BurchillAH@cf.ac.uk ${ }^{\dagger}$ University of Oxford,UK-dan.rogers@eng.ox.ac.uk
}

Keywords: hosting capacity, visualisation, distributed generation, distribution network, soft open points

\begin{abstract}
This paper presents an algorithm that calculates the nonconcurrent per-node demand and generation hosting capacity of a distribution network. The algorithm is used to appraise the increase in hosting capacity that would result from adding a Soft Open Point (SOP) to link two networks. To visualise non-concurrent per-node hosting capacity, a stress majorization technique is applied to a United Kingdom Generic Distribution System (UKGDS) network. This creates geographically representative layouts which preserve branch length information. It is suggested that applying these techniques to the entire GB $33 \mathrm{kV}$ network, whilst allowing for network reconfiguration and for variation of demand and generation across the time of day and day of year, is feasible.
\end{abstract}

\section{Introduction}

A tool to systematically evaluate the capacity available for connection of generation on the distribution network would be valuable. Presently, to gather information, prospective generators must submit requests to the Distribution Network Operators (DNOs). The response time and the type of information provided by the DNO varies [1]. A per-node hosting capacity assessment tool, of the sort presented in this paper, would help make the information available to prospective generators more consistent and allow fairer competition in the assessment of investment value.

In addition, as power-electronics based power flow control devices are developed for distribution network applications, DNOs will require methods to quantify and compare the impact of their deployment [2]. Of interest are back-to-back voltage source converter systems used to transfer power between normally separate parts of the network - these are referred to as Soft Normally Open Points (SNOPs) [3] or Soft Open Points (SOPs) [4] in the literature.

Hosting capacity is defined as the capacity of an electricity network to support distributed generation without violating pre-defined operating limits (e.g. maximum steady state bus voltage, line thermal limits) [5]. In one study, Bollen and Hassan [6] assess the hosting capacity for a prospective generator connected at different points on the network. These values can be thought of as the "per-node non-concurrent hosting capacity' in the sense that as soon as a new generator takes the capacity at one node, the hosting capacity at its neighbouring nodes is likely to change.

The mean per-node non-concurrent hosting capacity for a distinct area of network (e.g. one separated by normally open points and transformers) is useful in assessing the merit of techniques to increase network capacity, such as the addition of SOPs. Furthermore, if this methodology was to be applied to the entire GB distribution network on per-node basis, this would assist in the consistent provision of information to prospective generators or, if applied in the same way to demand, loads.

\section{Hosting Capacity Assessment}

\subsection{Definition of per-node non-concurrent hosting capacity}

The per-node non-concurrent generation (or demand) hosting capacity, $\mathbf{h}$, is the maximum generation (or demand), for each node, that can be supported without voltage, current or fault level exceeding limits anywhere within the network. This is expressed in Equation (1) where $g_{j}$ is additional real power generation (or demand) capacity at node $j$, where $N$ is the number of nodes in the network and $B$ is the number of branches (lines, cables and transformers) between nodes:

$$
\begin{gathered}
\mathbf{h}=\left[h_{1}, h_{2}, \ldots, h_{N}\right] \text { where } \\
h_{j}=\max \left(g_{j}\right) \text { subject to } \\
V_{i}^{\min } \leq V_{i} \leq V_{i}^{\max } \text { for } i=1,2, \ldots, N \\
F_{i}^{\min } \leq F_{i} \leq F_{i}^{\max } \text { for } i=1,2, \ldots, N \\
\left|I_{n}\right| \leq I_{n}^{\max } \text { for } i=1,2, \ldots, B
\end{gathered}
$$

Every node has an associated voltage and fault level minimum limit $\left(V_{i}^{\text {min }}\right.$ and $\left.F_{i}^{\text {min }}\right)$ and maximum limit $\left(V_{i}^{\max }\right.$ and $\left.F_{i}^{\max }\right)$ and every branch has an associated current magnitude limit $\left(I_{i}^{\max }\right)$.

\subsection{Application of the per-node non-concurrent hosting capacity approach}

Hosting capacity assessment was carried out on the $33 \mathrm{kV}$ nodes within the United Kingdom Generic Distribution System (UKGDS) EHV1 network using the default demand 
and generation values [7]. For reference, the node labels are shown in Figure 1.

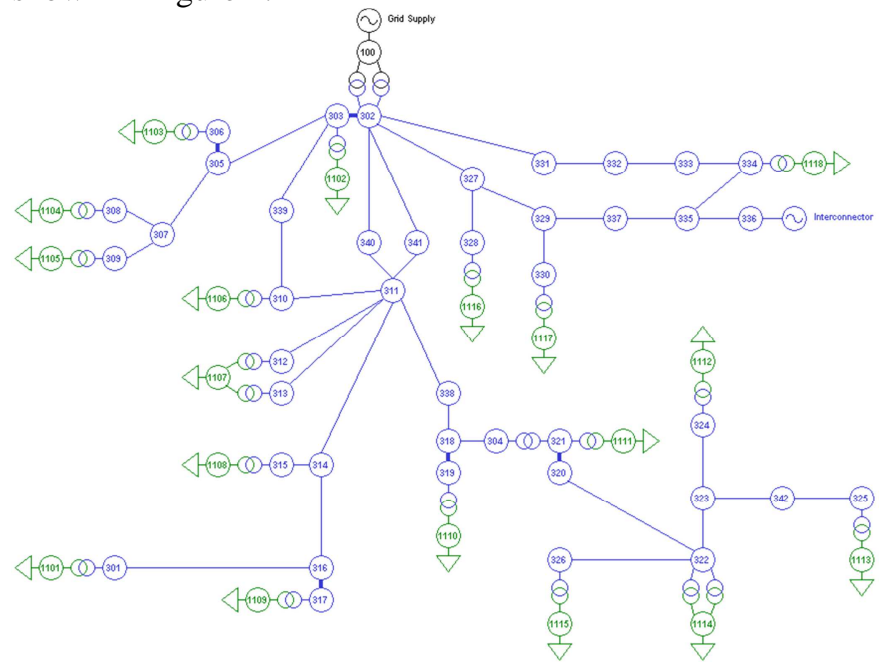

Figure 1. The UKGDS-EHV1 network. (Black $=132 \mathrm{kV}$, Blue $=33 \mathrm{kV}$, Green $=11 \mathrm{kV}$ )

The simulations were performed using OpenDSS with the "Newton" method [8]. Fault level calculations were not performed due to time constraints. The process used for obtaining the generation hosting capacity is shown in Figure 2. This returns an approximation (to the nearest $0.5 \mathrm{MW}$ ) of $\mathbf{h}$ from Equation (1). A similar approach was used to find the demand hosting capacity; the demand was incremented rather than the generation. The $132 / 33 \mathrm{kV}$ transformer tap changers were set to control the voltage at bus 301(a distant bus) within the range 0.9 to $1.1 \mathrm{pu}$. The voltage source at bus 336 was not included.

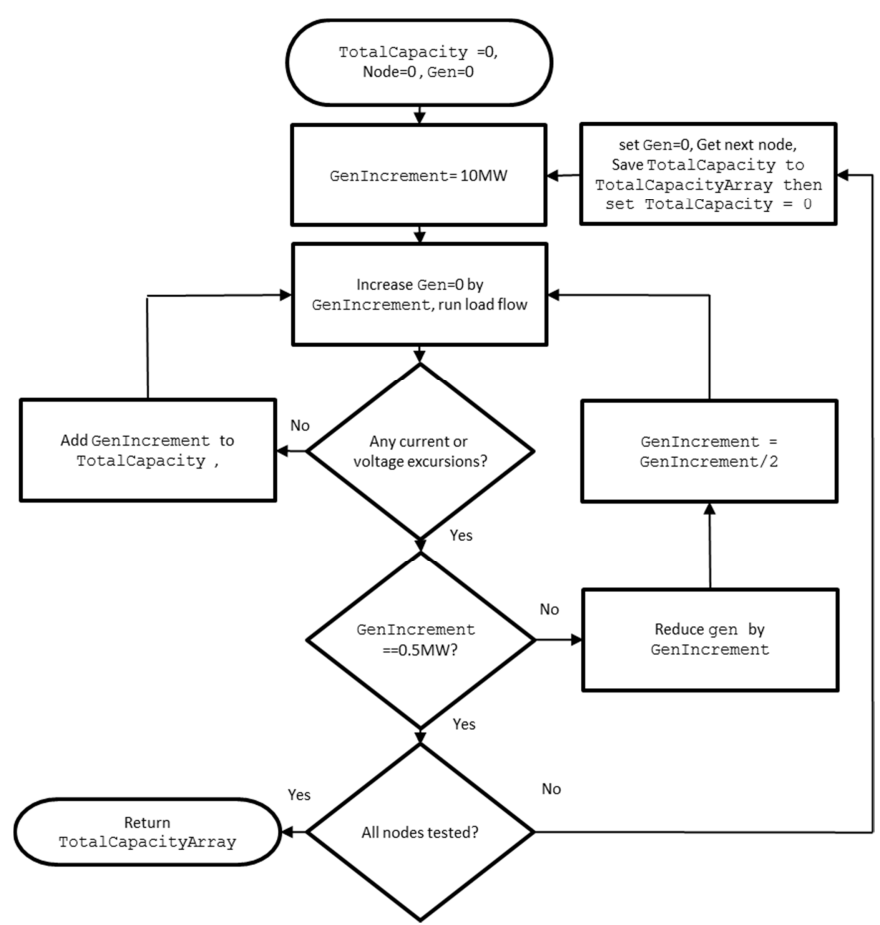

Figure 2. Process used to obtain per-node non-concurrent hosting capacity
The results are shown in a bar chart in Figure 3 and are visualised as a geographic layout in Figures 4 and 5. The diameter of each circle is proportional to the calculated hosting capacity at that node. The mean load capacity per node is $15.4 \mathrm{MW}$ and the mean generation capacity per-node is $23.0 \mathrm{MW}$.

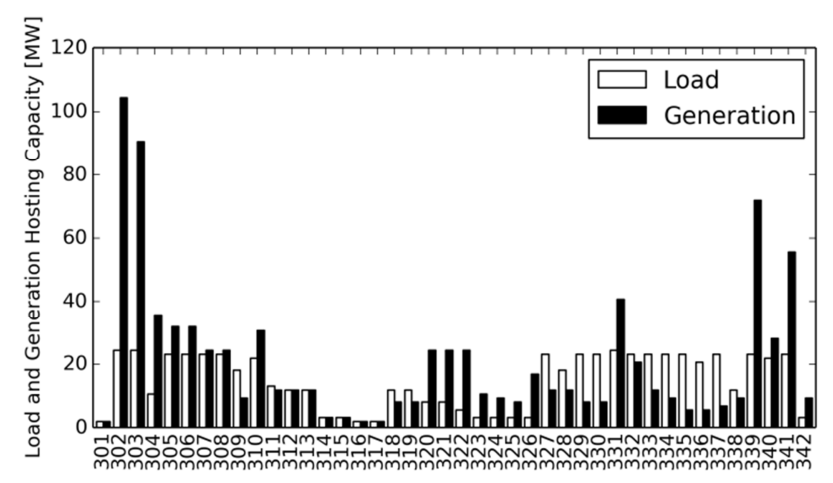

Figure 3. Per-node, non-concurrent hosting capacity assessment for the UKGDS-EHV1 network.

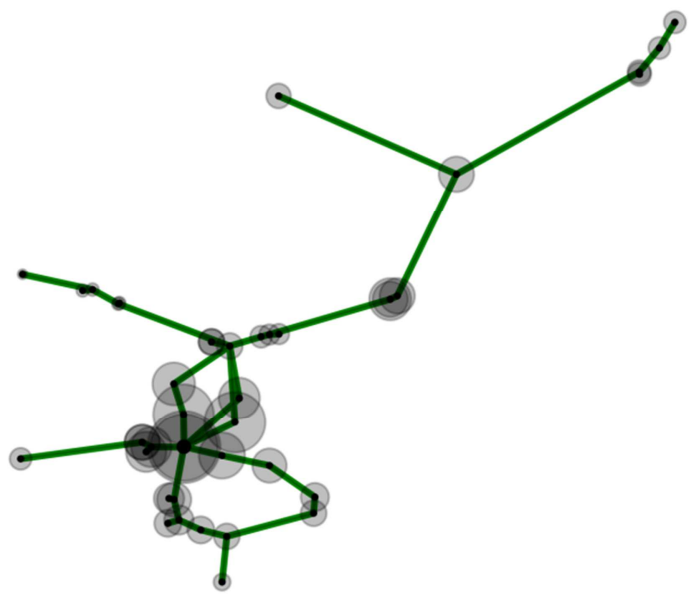

Figure 4. Geographical representation of UKGDS-EHV1 network with per-node generation hosting capacities.

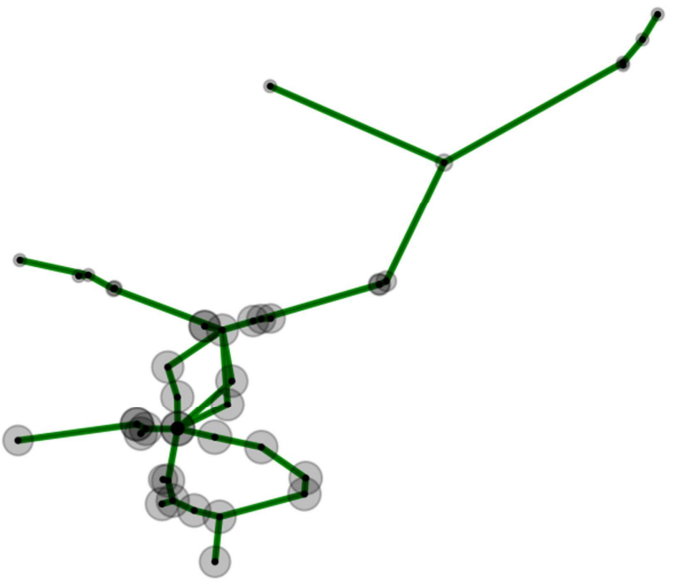

Figure 5. Geographical representation of UKGDS-EHV1 network with per-node demand hosting capacities. 
The geographically representative layouts, shown in Figures 4 and 5, were created using stress majorization [9]. This has the advantage, when compared to other graph layout algorithms, that the branch distance information is retained. Code for this, and for converting the UKGDS data to NetworkX [10] graph format, was written [11] using existing libraries [12]-[17]. Hosting capacity maps of this form would allow DNOs to present consistent information to prospective generators whilst also allowing them to withhold information on other loads or generators (e.g. if such disclosure was deemed not appropriate).

\section{Assessment of SOP benefits}

Hosting capacity assessment was used to quantify the potential benefit of adding an SOP between two distinct $33 \mathrm{kV}$ networks. Two UKGDS-EHV1 network models were used with an SOP connected between node 323 of the first network (network1) and node 336 of the second (network2). A line rated back-to-back converter was modelled as two adjacent PQ buses, as in [18]. One side of the SOP was represented as a load, the other as a generator, with the real power values set equal and the $\mathrm{Q}$ values independent as shown in Figure 6. When the direction of real power flow changes, the load and generator buses swap the network to which they are connected.

Per-node hosting capacity assessment was performed for a range of SOP real power (15 to $-15 \mathrm{MW}$ ) and reactive power (15 to -15 Mvar). A grid of $21 \times 21$ operating points was laid out (1.5 MW/Mvar resolution). The operating points where the apparent power is greater than 15 MVA were ignored; therefore a total of 317 SOP operating points per converter (i.e. per network) were assessed. The resulting changes in mean per-node generation and demand hosting capacity for each operating point are shown in the diagrams of Figure 8.

The coloured areas of Figure 8 indicate the permissible operating regions for each converter. The grey areas indicate the operating points at which connection of the SOP will cause voltage or currents to go outside limits. Green areas indicate an increase in mean hosting capacity and red areas indicate a reduction. A single operating point from this Figure 8 (indicated by a star, $\mathrm{P}=+/-4.5 \mathrm{MW}, \mathrm{Q} 1=\mathrm{Q} 2=4.5 \mathrm{Mvar}$ ), was selected and is examined in detail in Figure 9.

The selected operating point resulted in an increase in mean non-concurrent generation hosting capacity in network 1 of 9.0 MW/node and in network 2 of $1.8 \mathrm{MW} /$ node. An increase in mean non-concurrent demand hosting capacity of 1.7 MW/node (network 1) and a decrease of $3.3 \mathrm{MW} /$ node (network 2) was observed. These changes in hosting capacity are with reference to the case with no SOP (Figure 3 ).

For any operating point, the changes in mean per-node hosting capacity are not uniform across the nodes. Figure 9 shows the changes in node hosting capacity for the selected converter operating point. For each possible SOP operating point, even in the case of an increase in mean hosting capacity, some nodes show a decrease in hosting capacity. The maximum possible increase in capacity, from all possible SOP operating points, for every node, is shown in Figure 7.

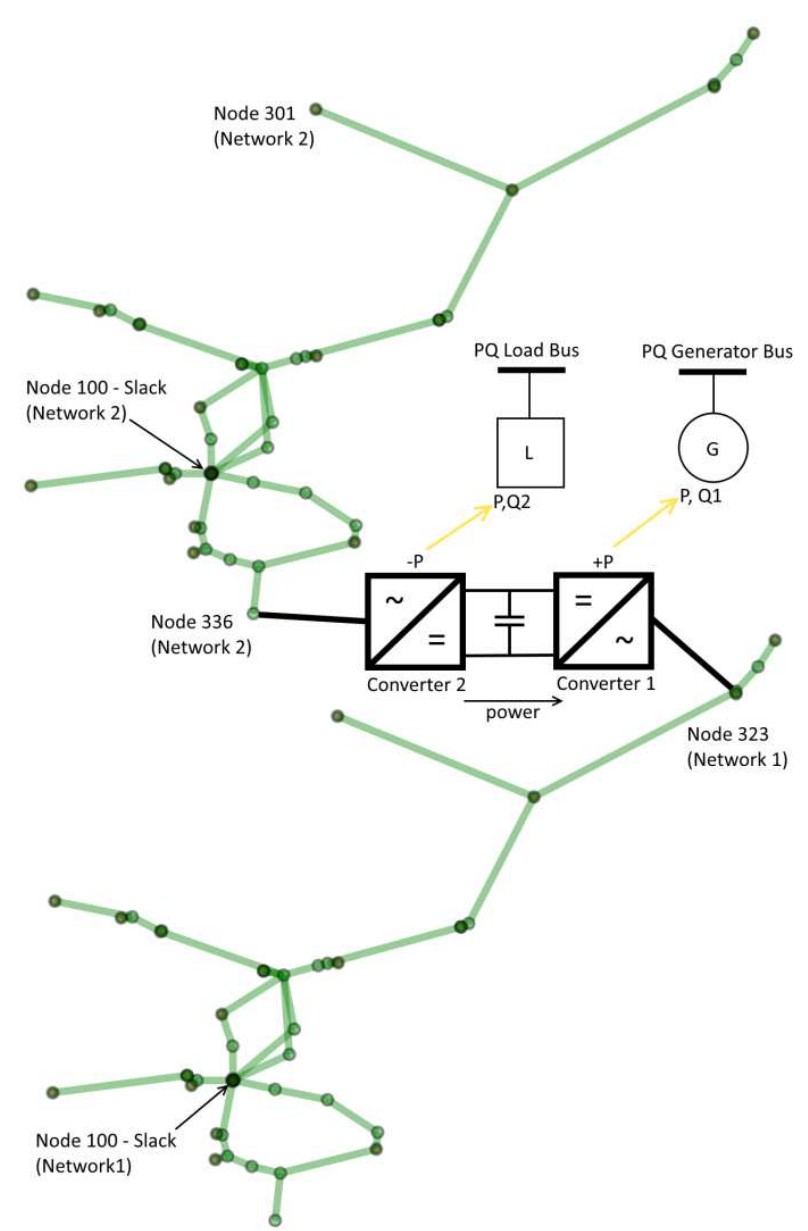

Figure 6. Back-to-back SOP connected between two networks
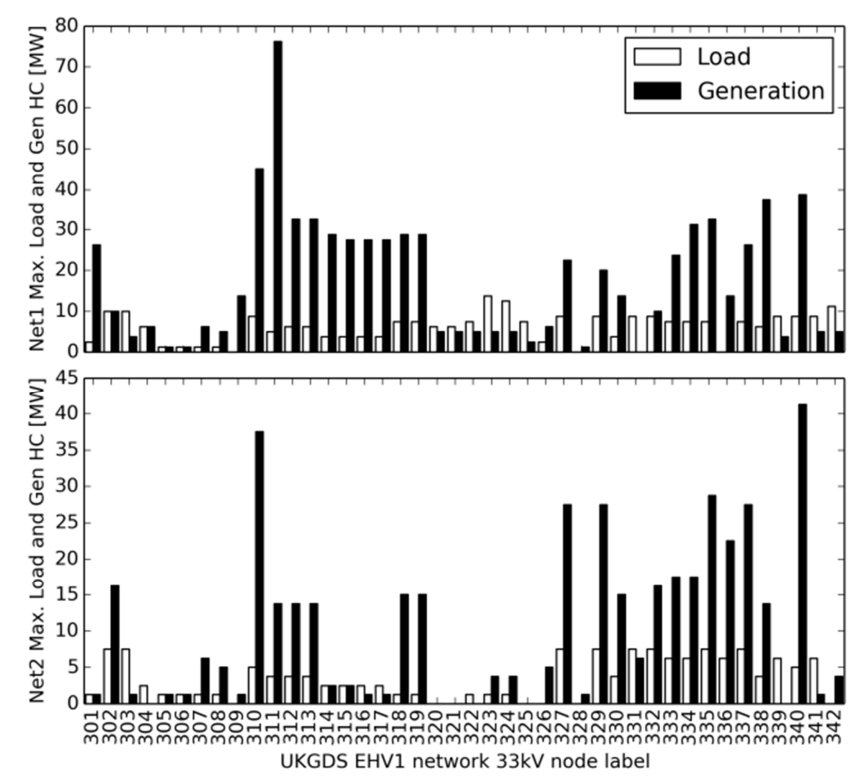

Figure 7. Maximum possible increase in hosting capacity after the addition of an SOP 


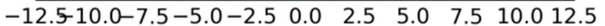

Converter1 Operating Points - Network1 Load

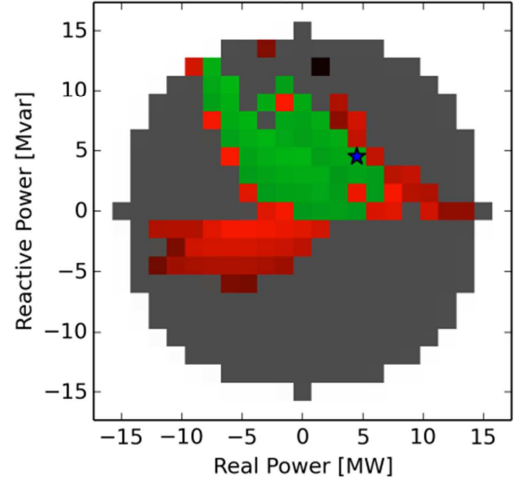

Converter1 Operating Points - Network1 Generation

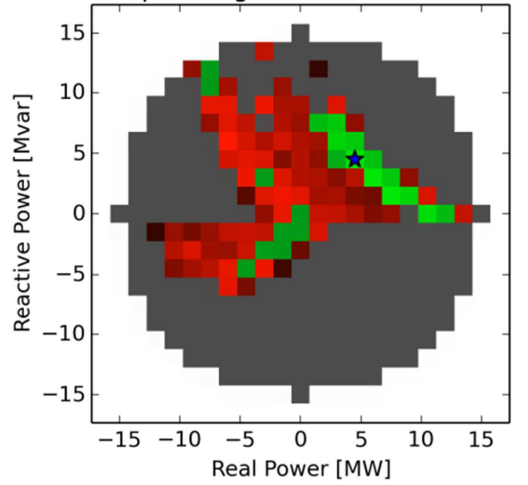

Converter2 Operating Points - Network2 Load

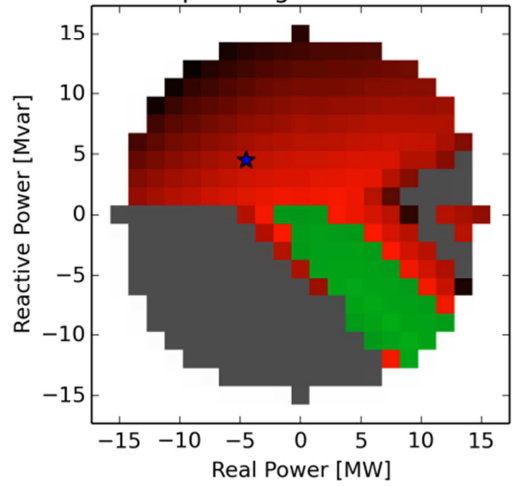

Converter2 Operating Points - Network2 Generation

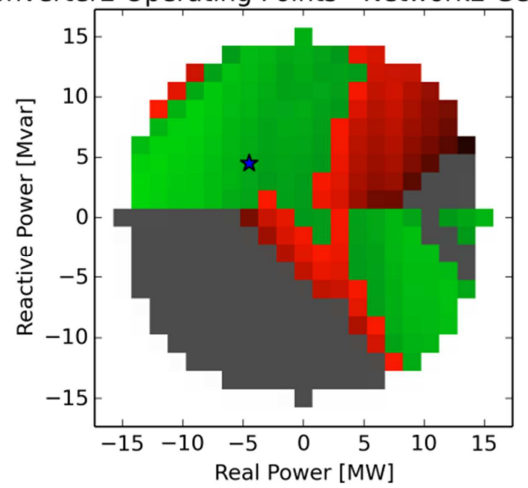

Figure 8. Change in mean non-concurrent hosting capacity for all converter operating points [MW]. Star indicates operating point $\mathrm{P}=+/-4.5 \mathrm{MW}, \mathrm{Q} 1=4.5 \mathrm{Mvar}, \mathrm{Q} 2=4.5 \mathrm{Mvar}$.
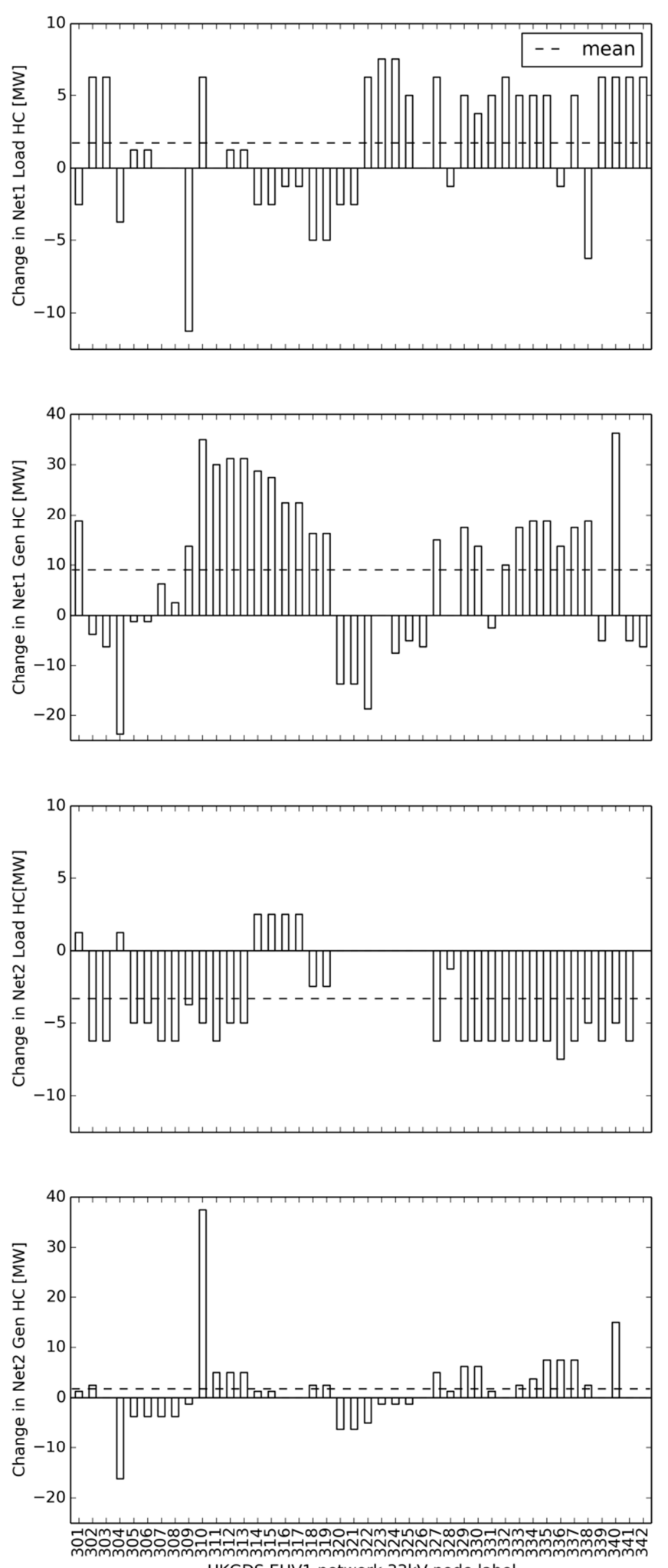

UKGDS EHV1 network 33kV node label

Figure 9. Change in non-concurrent hosting capacity $(\mathrm{HC})$ [MW] for operating points $\mathrm{P}=+/-4.5 \mathrm{MW}, \mathrm{Q} 1=4.5 \mathrm{Mvar}$, Q2=4.5 Mvar. 


\section{Application to the entire GB $33 \mathrm{kV}$ system}

For the results in section 3, a total of 227685 load flow calculations were performed at a rate of 9.6 load flow calculations per second. This was done on an Intel i5 processor, with 2GB RAM, running 32-bit Windows7. An overall CPU utilisation of approximately $25 \%$ was observed, with just two cores active. The mean time required to perform each capacity assessment was approximately 40 seconds. Using Equation (2) and the assumptions in Table 1, it would take approximately 400 days to assess the entire GB $33 \mathrm{kV}$ network (without the addition of SOPs).

Required number of hosting capacity assessments = NoDistinctNetworks $\times$ NoDistinctArrangementsPerNetwork $\times$ NoTimeStepsPerDay $\times$ NoDays

A reduction in the time taken is possible through optimising the code. To assess the potential for this, the number of floating point operations required to perform the hosting capacity assessment was assessed. Equation (3) shows a formula to approximate the number of floating point operations required to undertake a per-node hosting capacity assessment.
Number of Floating Point Operations for Hosting Capacity assessment $=$ NoDistinctNetworks $\times$ NoDistinctArrangementsPerNetwork $\times$ NoTimeStepsPerDay $\times$ NoDays $\times$ NoNodesPerNetwork $\times$ NoLF\&FaultCalcs $\times$ (NoFlopPerLF + NoFlopPerFaultCalc)

The number of floating point operations required to assess the entire GB $33 \mathrm{kV}$ network was estimated, using Equation (3) and the values in Table 1 , as $440 \times 10^{12}$ ( 2 sig. fig.). For reference, Cardiff University's Raven supercomputer achieves a peak of 42.6 TFlop/s. Practically, after implementation, $<10 \%$ of this would be achieved [19]. At these rates, the entire GB $33 \mathrm{kV}$ system could be analysed in a timeframe of the order of minutes.

If hosting capacity appraisal for the addition of a single SOP to each distinct $33 \mathrm{kV}$ network were to be carried out, as in section 3 (with 317 runs per converter), then $140 \times 10^{15}$ ( 2 sig. fig.) floating point operations would be required. This would require approximately 9 hours using the Raven supercomputer and therefore implies that the problem is tractable.

\begin{tabular}{|c|c|c|}
\hline Parameter & Estimate & Definition and source of estimate \\
\hline NoDistinctNetworks & 252 & $\begin{array}{l}\text { The number of distinct networks on which the hosting capacity assessment is } \\
\text { made. Estimated as the mean number of distinct } 33 \mathrm{kV} \text { networks (with their own } \\
\text { Bulk Supply Point and separated by Normally Open Points - NOPs) from a DNO } \\
\text { license area multiplied by the total number of license areas ( } 18 \times 14) \text {. }\end{array}$ \\
\hline NoDistinctArrangementsPerNetwork & 3 & $\begin{array}{l}\text { Number of ways that each network can be re-configured, including closing of } \\
\text { NOPs. Estimate of } 3 \text { is based on appraisal of a DNO's network. }\end{array}$ \\
\hline NoTimeStepsPerDay & 48 & $\begin{array}{l}\text { Number of time steps (for demand, generation and source voltage inputs). Half } \\
\text { hourly data assumed. }\end{array}$ \\
\hline NoDays & 24 & $\begin{array}{l}\text { Number of days in the year that assessment is run (to account for seasonal } \\
\text { variation in demand/generation). Assumed that two days (weekend and weekday) } \\
\text { in each month }(2 \times 12=24) \text { are used. }\end{array}$ \\
\hline NoNodesPerNetwork & 28 & $\begin{array}{l}\text { The mean number of nodes per distinct network. Sampled from a DNO's network } \\
\text { (a single license area). }\end{array}$ \\
\hline NoLFops & 9 & $\begin{array}{l}\text { Mean number of load flow operations from experimental runs on a DNO's } \\
\text { network. With 10MW starting increment finding hosting capacity to nearest } \\
0.5 \mathrm{MW} \text {. }\end{array}$ \\
\hline NoFlopPerLF & 1000000 & $\begin{array}{l}\text { Number of floating point calculations required per load flow operation. From } \\
\text { Zimmerman's [20] value for the Newton-Raphson algorithm with a } 125 \text { bus } \\
\text { system. It is assumed that conditional testing, file storage and other overheads are } \\
\text { not significant. It is assumed that the load flow calculation time scales linearly } \\
\text { with the number of nodes, as implied by [21]. }\end{array}$ \\
\hline NoFlopPerFaultCalc & 1000000 & $\begin{array}{l}\text { Number of floating point operations required per fault level calculation. This } \\
\text { assumes that a fault calculation could be performed in the same number of } \\
\text { floating point operations as the load flow operation. }\end{array}$ \\
\hline
\end{tabular}

Table 1: Definitions of parameters in Equation (3). 


\section{Conclusions}

The hosting capacity assessment approach presented in this paper provides a way of quantifying the benefit of power electronic Soft Open Points. A way of presenting changes in network hosting capacity, with varied operating parameters of a back to back power electronic soft open point, was introduced. The proposed method allows the network planner to survey the impact of each operating point of every node in the connected networks.

Assessment of the hosting capacity for every node on the entire GB $33 \mathrm{kV}$ network, each with a single SOP connection to a neighbouring network, is tractable. An estimated $140 \mathrm{x}$ $10^{15}$ floating point operations would be required, allowing for variation in network configuration and time-of-day/day-ofyear variation in demand and generation. If implemented, this would allow the provision of consistent information to prospective generators seeking to connect to the distribution network in Great Britain.

Existing graphing layout algorithms allow the visualisation of power network data without geographical information. An existing stress majorization layout algorithm was used to plot a UKGDS network. This has the advantage that, unlike many available algorithms, branch length information is taken into account. The resulting layout was used to demonstrate a means of visualising per-node non-concurrent hosting capacity. This is of benefit to research seeking to utilise network data without geographical co-ordinates, such as the UKGDS dataset.

\section{Acknowledgements}

This research was conducted under the EPSRC Reconfigurable Distribution Networks project (EP/K036327/1). The researchers would like to acknowledge and thank the funders.

\section{References}

[1] 'Energy Networks Association - Distributed Generation Forum', Cardiff, 23-Sep-2015.

[2] J. M. Bloemink and T. C. Green, 'Benefits of distribution-level power electronics for supporting distributed generation growth', IEEE Transactions on Power Delivery, vol. 28, no. 2, pp. 911-919, 2013.

[3] J. M. Bloemink and T. C. Green, 'Increasing distributed generation penetration using soft normally-open points', IEEE PES General Meeting, PES 2010, pp. 1-8, 2010.

[4] W. Cao, J. Wu, N. Jenkins, C. Wang, and T. Green, 'Operating principle of Soft Open Points for electrical distribution network operation', Applied Energy, vol. 164, pp. 245-257, Feb. 2016.

[5] N. Etherden and M. H. J. Bollen, 'Increasing the hosting capacity of distribution networks by curtailment of renewable energy resources', in 2011 IEEE Trondheim PowerTech, 2011, pp. 1-7.
[6] M. H. Bollen and F. Hassan, Integration of Distributed Generation in the Power System. John Wiley \& Sons, 2011.

[7] 'United Kingdom Generic Distribution System (UKGDS)'. [Online]. Available: http://www.sedg.ac.uk/.

[8] R. C. Dugan and T. E. McDermott, 'An open source platform for collaborating on smart grid research', in Power and Energy Society General Meeting, 2011 IEEE, 2011, pp. 1-7.

[9] E. R. Gansner, Y. Koren, and S. North, 'Graph Drawing by Stress Majorization', in Graph Drawing: 12th International Symposium, GD 2004, New York, NY, USA, September 29-October 2, 2004, Revised Selected Papers, J. Pach, Ed. Berlin, Heidelberg: Springer Berlin Heidelberg, 2005, pp. 239-250.

[10] A. A. Hagberg, D. A. Schult, and P. J. Swart, 'Exploring Network Structure, Dynamics, and Function using NetworkX', in Proceedings of the 7th Python in Science conference (SciPy 2008), pp. 11-15.

[11] L. J. Thomas, 'ukgds2nx: UK Generic Distribution Network to NetworkX converter and grapher.' [Online]. Available: https://github.com/4c656554/ukgds2nx. [Accessed: 21-Apr-2016].

[12] S. Van Der Walt, S. C. Colbert, and G. Varoquaux, 'The NumPy array: a structure for efficient numerical computation', Computing in Science \& Engineering, vol. 13 , no. 2, pp. 22-30, 2011.

[13] W. McKinney, 'Data structures for statistical computing in Python', in Proceedings of the 9th Python in Science Conference, 2010, vol. 445, pp. 51-56.

[14] E. R. Gansner and S. C. North, 'An open graph visualization system and its applications to software engineering', Software - Practice and Experience, vol. 30, no. 11, pp. 1203-1233, 2000.

[15] J. D. Hunter, 'Matplotlib: A 2D graphics environment', Computing in science and engineering, vol. 9, no. 3, pp. 90-95, 2007.

[16] E. Jones, T. Oliphant, and P. Peterson, 'SciPy: Open Source Scientific Tools for Python, 2001'. [Online]. Available: http://www.scipy.org/.

[17] A. A. Hagberg, D. A. Schult, and M. Renieris, 'PyGraphviz - Python interface to the Graphviz graph layout and visualization package'. [Online]. Available: http://pygraphviz.github.io/.

[18] W. Cao, J. Wu, N. Jenkins, C. Wang, and T. Green, 'Benefits analysis of Soft Open Points for electrical distribution network operation', Applied Energy, vol. 165, pp. 36-47, Mar. 2016.

[19] J. M. Bull, 'Single Node Performance Analysis of Applications on HPCx', UK National Supercomputing Service 2002 - 2010, HPCxTR0703, 2007.

[20] R. D. Zimmerman, 'Comprehensive distribution power flow: modeling, formulation, solution algorithms and analysis - PhD Thesis', 1995.

[21] M. Marc-André, S. Frédéric, and B. David, 'Review of Open Source Power Grid Simulation Tools for LongTerm Parametric Simulations', Natural Resources Canada, 2011-137 (RP-TEC) 411-MODSIM, Jul. 2011. 